\title{
O CUIDADO HUMANO TRANSICIONAL NA TRAJETÓRIA DE ENFERMAGEM
}

Ivete Palmira Sanson Zagonel*

ZAGONEL, I.P.S. O cuidado humano transicional na trajetória de enfermagem. Rev.latino-am.enfermagem, Ribeirão Preto, v. 7, n. 3, p. 25-32, julho 1999.

O presente trabalho aborda os eventos transicionais do ser humano que permeiam o ciclo vital, bem como as condições condutivas aos processos de transição e suas relações ao cuidado. Enfoca a distinção entre os vários tipos de transição e suas conceituações dentro da dimensão de cuidado transicional. O cuidado considera as características dos eventos que produzem a mudança, bem como variáveis individuais e de contexto que interagem com as características do evento, facilita a compreensão do processo de interação e assimilação da mudança. A partir dessa contextualização aponta para o desenvolvimento de intervenções de enfermagem diante dos processos transicionais pela utilização de teorias de enfermagem, as quais podem ser reafirmadas em termos de transição. Pela variedade de enfoques a transição relaciona-se ao cuidado de enfermagem, visa a prevenção e intervenção para cada caso específico de ocorrência, possibilita aos enfermeiros um enfoque inovador de cuidado. Aponta que o cuidado está ligado de alguma forma a cada estágio desenvolvimental, favorece a maturidade e crescimento em busca de maior equilibrio e estabilidade. O cuidado diante da transição traz respostas à valorização do ser.

UNITERMOS: processos grupais, cuidado de enfermagem

\section{INTRODUÇÃO}

O ciclo de vida individual acontece dentro do ciclo de vida familiar que é o contexto primário do desenvolvimento humano. Esta perspectiva aponta para a ênfase na compreensão dos problemas que as pessoas desenvolvem à medida que se movimentam juntas através da vida. Este movimento é mudança, processo, resultado, direção dos padrões vitais fundamentais do ser humano. Esta mudança que ocorre a nível individual e familiar chama-se transição. Para SCHUMACHER \& MELEIS (1994), transição é um conceito de interesse em enfermagem, é um "conceito central” a pesquisadores, assistenciais e teóricos uma vez que está se presenciando um novo paradigma. São mudanças muitas vezes dramáticas que estão atraindo a atenção para que os profissionais, especialmente o enfermeiro, desenvolvam novas perspectivas, reflexão e criatividade para a realidade presente, conduzindo a uma revisão na mentalidade e nos valores sociais. Nos pontos de transição de um estágio para outro no processo de desenvolvimento familiar é que ocorrem os maiores geradores de stress, principalmente nos momentos de grande instabilidade, insegurança, rupturas do ciclo vital. Nestes pontos vulneráveis é que os esforços devem ser intensificados no sentido de reorganizar os momentos transicionais.

As condições condutivas aos processos de transição estão basicamente ligadas a três tipos de transição: a desenvolvimental, a situacional e de saúdedoença, as quais, podem gerar instabilidade produzindo efeitos negativos e profundas alterações, passageiras ou permanentes, deixando a sua marca no indivíduo. A relação enfermeiro-cliente freqüentemente ocorre durante períodos de instabilidade em qualquer destes três níveis citados.

Em seu artigo, CHICK \& MELEIS (1986, p. 239) definiram transição como "uma passagem ou movimento de um estado, condição ou lugar para outro", dizem que a transição tem conotação com o tempo e o movimento. Pode-se considerar que a vida em si constitui uma transição. O complexo ciclo vital é um constante transformar-se de um estado, fase ou períodos para outro. Eventos marcantes são referidos por CARTER \& McGOLDRICK (1995) como casamento, nascimento e educação dos filhos, saída dos filhos do lar, aposentadoria e morte. Estes eventos apresentam subeventos que vão sendo delineados durante a evolução de cada fase. HALL (1981, p. 2) chama de "paradigma de mudança" a estes

* Enfermeira, Professora Adjunta do Departamento de Enfermagem da UFPr. Doutoranda do Curso de Doutorado em Filosofia de Enfermagem da UFSC, Membro do Programa Integrado de Pesquisa Cuidando e Confortando (PIP C\&C), coordenado pela Prof ${ }^{a}$ $\mathrm{Dr}^{\mathrm{a}}$ Eloita Neves 
estágios vitais, em que os "fenômenos são vistos como se eles estivessem em estados contínuos de transição". Durante a evolução humana a mudança é inevitável mas não necessariamente natural ou desejável. A este respeito, CHICK \& MELEIS (1986) enfatizam que a transição é iniciada, em alguns casos, por eventos que vão além do controle individual e em outros ela pode ser procurada deliberadamente através de eventos tais como: casamento, gravidez, mudança de profissão ou cirurgia.

A enfermagem, ao atuar nestas circunstâncias, desenvolve o cuidado transicional em um duplo movimento, em que os processos de transição geram alterações de saúde-doença e estes levam à transições. Em qualquer das situações a intervenção de enfermagem deve estar presente.

Algumas teorias de enfermagem como de Peplau, Travelbee, Roy, Rogers, Newman e Orem que enfocam, respectivamente, o crescimento e desenvolvimento pessoal, o encontro de significado à experiência da doença, a adaptação, a organização unitária emergente da pessoa, a movimentação do ser humano em contínua e permanente mudança, modificações na capacidade de autocuidado, fornecem estratégias de cuidado objetivando à manutenção da saúde ou metas de promoção à saúde, com vistas a esses eventos.

Este trabalho propõe-se a delinear o conceito de transição, sua utilização nos vários campos de enfermagem, situar o cuidado em eventos de transição, algumas teorias que adaptam-se à transição, visando o alcance de um processo transicional saudável. E uma reflexão tentativa no sentido de despertar no enfermeiro uma prática de cuidados voltada aos aspectos que permeiam os eventos de transição no ciclo vital, fazendo ligações teóricas e empíricas para enriquecer o conhecimento de enfermagem.

\section{CONCEITUALIZAÇÃO DE TRANSIÇÃO}

Em uma perspectiva de transição para a prática de enfermagem mais humanizada, científica, compartilhada, holística, se requer um conhecimento a respeito do crescimento e desenvolvimento ao longo do ciclo vital, enfrentamentos e adaptações aos eventos importantes da vida e mudanças no estado de saúde de um estágio de bem estar para um de enfermidade. Essa instabilidade transicional é desencadeada por mudanças desenvolvimentais, situacionais ou de saúde-doença (SCHUMACHER \& MELEIS, 1994; CHICK \& MELEIS, 1986; MURPHY, 1990).

Transição para HALL (1981) é um termo familiar às teorias de desenvolvimento, de stress e de adaptação. Está relacionada à mudança e desenvolvimento, acomodando processos vitais contínuos e descontínuos dos seres humanos. Em seu artigo CHICK \& MELEIS (1986) expõem claramente que as transições recaem no domínio da enfermagem quando elas são pertinentes à saúde ou doença ou quando as respostas à transição são manifestadas nos comportamentos relacionados à saúde. MURPHY (1990) refere que o enfermeiro pode atuar nos períodos antecipatórios, de preparação para a mudança de papéis, de prevenção dos efeitos negativos sobre o indivíduo, como exemplo, a preparação à maternidade. A enfermagem tem utilizado o tema transição como um de seus enfoques centrais, considerando este como períodos entre estados completamente estáveis. Isso é comprovado pela pesquisa realizada por SCHUMACHER \& MELEIS (1994) quando as autoras realizaram uma revisão teórica de artigos publicados de teoria, prática e pesquisa de enfermagem, no período de 1986 a 1992. Foram selecionados 310 artigos sempre que a palavra "transição" aparecesse no título. Pela riqueza de idéias e variedade dos escritos as autoras revelaram que a enfermagem tem utilizado a transição de várias maneiras e em diferentes situações, puderam expandir as categorias já apresentadas em artigo anterior de CHICK \& MELEIS (1986) e traçaram uma tipologia de transições para demonstrar a abrangência do fenômeno.

Em seu artigo de 1957, Tyhurst, citado por MURPHY (1990, p. 2), introduz o termo transição na literatura de saúde mental e o define a partir do verbo do latim "transire" ou atravessar, como "uma passagem ou mudança de um lugar, estado ou ato ou conjunto de circunstâncias para outro”. CHICK \& MELEIS (1986) chamam a transição de substantivo, com o mesmo significado do verbo.

Para MERCER et al. (1988, p. 144) as transições são definidas como "pontos de mudança os quais resultam no pressuposto de novos papéis e novas relações conduzindo-a a novas autoconcepções". Também consideram transições as mudanças nas atividades e papéis da vida externa e as mudanças na perspectiva e autodefinição da vida interna. Este conceito foi derivado de uma pesquisa realizada por estes autores, utilizando história de vida de mães e não mães, com idade entre 60 e 95 anos, para identificar as diferenças entre os dois grupos das transições de desenvolvimento ao longo do ciclo vital.

Muitos dos trabalhos sobre transições, afirmam SCHUMACHER \& MELEIS (1994), tem enfocado o indivíduo, porém é crescente o interesse de estudiosos em referir-se a transições de desenvolvimento, como por exemplo, a relação mãe-fillho, da família, enfermeirocliente. Com esta abordagem, situa-se o trabalho de TOMLINSON (1987) que estudou 96 casais para determinar as diferenças conjugais de satisfação marital durante a transição à paternidade. Os resultados deste 
estudo sugerem que a percepção materna da satisfação conjugal após a maternidade é mais complexa do que a do pai.

MURPHY (1990) refere que o conceito transição parece ser definido a partir de duas perspectivas teóricas. A primeira é o desenvolvimento ao longo da vida do indivíduo, no qual ocorrem eventos marcantes; a segunda os eventos de vida produzem desequilíbrio entre dois períodos de tempo estáveis. Estas perspectivas enfatizam as respostas humanas à transição. A extensão e a intensidade desta influência transicional variam ao longo do tempo, afetando de forma diferente os indivíduos que experienciam um mesmo evento. Os significados atribuídos a transições variam de pessoa a pessoa e, assim, influenciam o resultado das mesmas. CARTER \& McGOLDRICK (1995) vão mais além, englobam a família, como um sistema movendo-se através do tempo possuindo propriedades diferentes de outros sistemas, indicando que os significados de transições mudam de família a família bem como de família a sistema. Por exemplo, o nascimento de um primeiro filho força uma reorganização familiar como um sistema social. A este respeito MAJEWSKI (1986, p. 10) diz que “os papéis tem que ser remodelados, as posições de status mudadas, os valores reorientados e necessidades realizadas através de novos canais”. É essa transição à paternidade que confronta os casais e a própria família, uma vez que, como refere McGOLDRICK (1995), hoje as mulheres não vêem mais o casamento como um evento obrigatório para passar à fase adulta, e aquelas que optam em casarse, estão querendo a sua própria carreira e dividir as responsabilidades primárias pela casa e os filhos. Esta postura da mulher diante do casamento denota as mudanças a que está passando, gerando mudanças a nível familiar e à paternidade, exigindo uma nova remodelação de papéis.

A descrição de Bridges citado por MURPHY (1990), inclui três fases ao processo de transição: o término, caracterizado por várias características negativas, a reavaliação e novos inícios. Estas fases ocorrem como ritos, rituais ou cerimônias importantes. FRIEDMAN (1995, p. 144) chama de ritos de passagem naturais, a morte, o casamento, a adolescência. Ela começa pela morte por considerar "o mais importante evento isolado na vida familiar". É um evento importante associado a um membro da família e que pode influenciar outros eventos do ciclo vital.

Aos eventos de transições do ciclo de vida, IMBER-BLACK (1995) chama de normativos, como os casamentos, nascimentos e mortes e indica como transições idiossincráticas àquelas de natureza aparentemente diferente ou rara, não assinaladas por rituais, como o nascimento de um filho deficiente, abortamento, separação forçada através da hospitalização, suicídio, doença crônica incapacitante, entre outras.
Terkelson, citado por IMBER-BLACK (1995), acrescentou a categoria paranormativa, para incluir aquelas transições como a separação conjugal, doença e graves eventos extrínsecos e inesperados com os quais uma família deve lidar. A escassez de rituais que assinalam a mudança de desenvolvimento é encontrada em eventos como: falta de apoio social, rompimentos de relacionamentos e isolamento, estigma, segredo, sentimento de vergonha. Complementa referindo que os rituais ajudam a lidar com a mudança e não devem ser considerados uma ameaça.

Nos processos de mudanças as transições passam por estabilidade - instabilidade e estabilidade como um fenômeno interligado ao movimento e fluxo, caracterizado pela entrada, passagem e saída. (MURPHY, 1990; CHICK \& MELEIS, 1986). Percebe-se que apesar da diversidade das transições, algumas semelhanças podem ser percebidas. A estas similaridades SCHUMACHER \& MELEIS (1994, p. 121) denominaram de "propriedades universais de transições". Dentre as propriedades incluem-se as transições como processos que ocorrem ao longo do tempo, envolvendo desenvolvimento, fluxo e movimento e também a natureza da mudança que permeia a vida dos indivíduos e famílias, durante a transição.

As transições são acompanhadas por uma ampla gama de emoções, muitas das quais surgidas pelas dificuldades encontradas durante a transição. Além do aspecto emocional, o bem estar físico é também importante, uma vez que o desconforto físico acompanha uma transição e pode interferir em uma passagem bem sucedida. Considerando estes aspectos é que surge o cuidado de enfermagem, voltado a uma maior sensibilização, conscientização e humanização, identificando no cliente fatores que indiquem a transição, com a finalidade de facilitar estes eventos em direção à uma transição saudável, emergindo, assim, o cuidado transicional.

\section{A DIMENSÃO DO CUIDADO TRANSICIONAL}

Certos aspectos da vida de um indivíduo são afetados mais do que outros pela transição que ele experiência e a extensão e intensidade desta influência podem variar ao longo do tempo. As transições podem estar ligadas aos estágios da idade, aos eventos inesperados, às experiências de doença, às carreiras profissionais, todas com íntima relação com o ambiente. As respostas de quem está passando por transições são variadas incluindo aspectos internos e externos que circundam este momento.

CHICK \& MELEIS (1986) consideram que a 
transição tem uma característica essencialmente positiva, uma vez que a pessoa, ao passar pelo evento, alcança uma maior maturidade e estabilidade ao que passou. $\mathrm{O}$ cuidado de enfermagem considerando esses indicadores se torna extremamente importante a partir do conhecimento de transição e suas conseqüências ao indivíduo em todas as circunstâncias biopsicosocioculturais.

Os pressupostos básicos que devem acompanhar as estratégias de cuidado realizadas pelo enfermeiro a um cliente em transição inserem-se na compreensão da transição a partir da perspectiva de quem a experiencia e identificação das necessidades para o cuidado com essa abordagem. Para MURPHY (1990) é necessário considerar os fatores que mediam os processos de transição, ou seja, os individuais, os ambientais e as terapêuticas de enfermagem e incluem as ações preventivas à transição e as estratégias de intervenção quando o evento já ocorreu ou está em andamento.

A transição será melhor sucedida ao conhecerse: o que desencadeia a mudança; a antecipação do evento; a preparação para mover-se dentro da mudança; a possibilidade de ocorrências múltiplas de transições simultaneamente. $\mathrm{O}$ enfoque está na disposição para ajudar na passagem de um estado a outro considerando que as situações difíceis irão gerar respostas positivas e negativas. É necessário lidar com os acréscimos e decréscimos da pessoa. As situações envolvidas com as transições são variadas e todas estão ligadas ao cuidado de enfermagem. Para se ter uma noção da abrangência da utilização, CHICK \& MELEIS (1986) indicam alguns eventos como a doença, recuperação, hospitalização, maturidade, gravidez, nascimento, imigração, migração, aposentadoria, perda e morte. MURPHY (1990) acrescenta a estes, a puberdade, casamento, gestação na adolescência.

Na revisão de literatura realizada sobre transição em enfermagem, SCHUMACHER \& MELEIS (1994) relatam a aplicabilidade no ensino, referindo as transições curriculares e educacionais, na profissão, mudanças de unidades de trabalho, turnos, equipes, entrada e saída do emprego e na sociedade, enfocando relacionamentos diádicos, familiares, transição à paternidade, o período pós-parto e os vários contextos de doença que exigem cuidados especiais pelas características da situação.

O cuidado humano vem sendo estudado e tem influenciado a teoria, pesquisa, prática e educação de enfermagem. MORSE et al. $(1990,1991)$ examinaram o conceito de cuidado entre 35 pesquisadores, que resultou na identificação de cinco perspectivas epistemológicas: cuidado como característica humana, como um imperativo moral ou ideal, como afeto, como relação interpessoal e como intervenção de enfermagem. E adicionais a estas, as autoras referem dois resultados do cuidado que foram identificados como experiência subjetiva e como resposta física do paciente.

O cuidado para GRIFFIN (1983) engloba as atividades e as atitudes/sentimentos. As atividades são definidas como assistir, ajudar e servir, mediadas através da relação enfermeiro-cliente que podem ser chamadas de cuidados se a expressão de emoções estiver complementando-as. Ao tornar-se um paciente, a pessoa perde temporariamente sua autonomia a qual pode levar a uma perda de dignidade. Nesta circunstância o cliente precisa ser moral e emocionalmente reconhecido naquilo que ele foi e espera ser para não prejudicar a sua identidade. Diz ainda que as atividades de cuidado são geradas pela percepção e julgamento do enfermeiro a cerca das necessidades do cliente em um dado período. O reconhecimento da importância do cliente como pessoa, percebendo os elementos emocionais, implica para a autora estar motivando e energizando o ato que chama de cuidado.

Similarmente DREW (1986) refere como cuidado, os termos confirmação e exclusão em um estudo que realizou explorando os aspectos positivos e negativos da relação de interação entre o cliente e os cuidadores. Esta pesquisa indica o quanto o cliente pode sentir diante de um evento de mudança e o quanto o enfermeiro pode não perceber se estiver voltado apenas ao cumprimento de suas tarefas competentemente técnicas.

ROACH (1993, p. 47) refere-se ao cuidado como um modo humano de ser, não reduzivel a ações específicas e inclui "a capacidade ou poder para cuidar, uma capacidade ligada e inseparável da nossa natureza, como seres humanos". Diz ainda que o cuidar, o ajudar tem sido a grande motivação de estudantes ao ingressarem na enfermagem nos Estados Unidos. Esta autora não tem mais utilizado o cuidado como um "atributo" humano essencial, por considerar que o termo tem interpretações diversas, limita e particulariza uma situação, não refletindo a natureza e essência do ser humano.

A literatura de enfermagem que aborda o cuidado é basicamente americana, está difundindo-se em outros países dentre os quais o Brasil, (WALDOW, 1995). PATRÍCIO (1992, 1993) faz uma reflexão no sentido de colaborar com a compreensão do conceito cuidado como um instrumento da enfermagem para promoção da cidadania e sobre uma experiência em saúde comunitária com um referencial enfocando o cuidado de caráter holístico; SILVA \& BORENSTEIN (1992) realizam uma reflexão do cuidar pesquisando com - o - Ser doente à luz de Heidegger; SANTOS (1993) revela o cuidado enquanto conceito central à enfermagem e comenta sobre sua dimensão de trabalho invisível; CASTANHEL \& BOEHS (1993) relatam a experiência de cuidar de uma família utilizando o referencial de Leininger. NEVESARRUDA \& SILVA (1994) expõem a criação de um programa de pesquisa para desenvolver conceitos e teorias de enfermagem sobre o cuidar/cuidado e confortar/ 
conforto, com um compromisso de transformar a realidade; NUNES \& NEVES-ARRUDA (1995) apresentam uma revisão de literatura sobre o significado de conforto e sua importância no processo multidimensional de cuidar. Outros autores de enfermagem tem se dedicado ao tema como BOEMER (1984); WALDOW (1992); RADÜNZ (1994); ERDMANN (1995); NUNES (1995); SOUZA (1995); SILVA (1996); LACERDA (1996).

Todos estes estudos abordam a variada gama de abrangência do cuidado e o quanto ele está internalizado no fazer e pensar diário da enfermagem atual, com enfoques de aplicação em eventos de situações, de desenvolvimento e de saúde - doença, onde cada autor introduz a sua visão de mundo, o seu referencial de vida, diante da transição. O termo transição não é utilizado explicitamente, mas observa-se a sua presença pela dimensão da aplicabilidade do cuidado em eventos transicionais do ser. Ao aplicar o cuidado diante de eventos de transição surge o "cuidado transicional". Como respostas ao evento de transição, originam-se comportamentos que para CHICK \& MELEIS (1986) podem ser observáveis ou não. Estas respostas englobam processos intra-psíquicos bem como o contexto sociocultural, referidos por MURPHY (1990, p. 241), como "desorientação, stress, irritabilidade, ansiedade, depressão, mudanças no autoconceito, mudanças no desempenho de papel, mudanças na auto-estima $e$ outros".

Conhecendo estes padrões o enfermeiro pode avaliar, planejar e implementar estratégias de prevenção, promoção e intervenção terapêutica frente aos eventos de transição, objetivando restabelecer a conexão e instabilidade geradas. Mesmo quando não mais couberem medidas preventivas, a avaliação para apontar as transições pessoais e sociais a que o cliente está envolvido deve ser executada. Uma das formas de se desenvolver intervenções de enfermagem diante dos processos transicionais é a utilização das teorias de enfermagem que podem ser reafirmadas em termos de transição.

\section{TEORIAS DE ENFERMAGEM E TRANSIÇÃO}

As teorias de enfermagem têm uma importante missão de inter-relacionar a teoria, pesquisa e prática, uma apoiando a outra. As teorias de enfermagem para CHICK \& MELEIS (1986) devem conceituar mudança de uma forma ou outra. TORRES (1986) diz que o conhecimento não existe de uma forma estática, pois estamos constantemente adicionando ao que conhecemos ou acreditamos ser verdadeiro. A principal abordagem dentro das teorias de enfermagem para TORRES (1986, p. 1) tem sido “a compreensão dos seres humanos dentro de seu ambiente em relação às suas necessidades". WATSON (1985, p.1) define teoria como "um agrupamento imaginativo do conhecimento, idéias $e$ experiências que são representadas simbolicamente e buscam esclarecer um dado fenômeno". Para a autora, a teoria fornece credibilidade às idéias e experiências pessoais dos enfermeiros, como valiosa fonte de conhecimento. Algumas teoristas deixam bem claro o tema de sua teoria, como é o caso de Nightingale com enfoque no ambiente ou de Henderson com ênfase nas necessidades. Outras podem interligar mais de um tema como Rogers e Hall.

Peplau, uma das primeiras teoristas que surge depois de Nightingale, refere-se ao processo interpessoal enfocando principalmente as necessidades psicológicas do cliente. Por ter sido publicada em 1952, CAREY et al. (1989), afirmam que sua teoria pode ser considerada uma das pioneiras no campo da enfermagem. Peplau considera que à medida que aumenta a interação enfermeiro-paciente aumenta a compreensão dos papéis mútuos em torno do problema. Ao compartilhar a interação, buscam a solução do problema, reconhecendo, esclarecendo e definindo o que gerou a situação estressante. A teoria de Peplau escrita há décadas continua muito atual e adapta-se aos eventos transicionais, por levar em consideração o evento ou situação que gerou a crise para juntos, enfermeiro e paciente, encontrarem mutuamente a melhor solução ao desequilíbrio.

Johnson, em 1968, propõe pela primeira vez conforme CONNER \& WATT (1989) seu modelo de cuidados de enfermagem, referindo que estes facilitam a manutenção de um estado de equilíbrio no cliente. Indicou que o stress pode advir de estímulos da natureza, internos e externos. Estes estímulos criam tensões gerando um estado de desequilíbrio. Conforme FOLI et al. (1989), Johnson considera a doença como agente que perturba a estabilidade e a obtenção de resultados positivos. Este enfoque teórico adapta-se ao que Chiriboga apud MURPHY (1990, p. 2) enfatiza, que “o curso da vida é marcado por períodos de estabilidade, instabilidade e retomada da estabilidade”. Johnson é consistente em sua teoria com o conceito de transição considerado como um período entre dois estados de equilíbrio. A sua obra explica quatro afirmações teóricas, conforme FOLI et al. (1989), de que a intervenção de enfermagem se baseia na conservação da energia, na integridade estrutural, pessoal e social de cada cliente. O homem luta constantemente para manter o equilíbrio e a teoria de Johnson fornece um suporte para as adaptações e ajustamentos necessários.

HOBBLE \& LANSINGER (1989), ao referiremse ao processo interpessoal de Travelbee, dizem que este está relacionado à transição uma vez que propõe a ajuda do enfermeiro ao indivíduo, família ou comunidade para 
evitar ou atuar diante da experiência de enfermidade, sofrimento, visando encontrar o sentido da própria experiência e como ser humano. Considera o indivíduo como em um contínuo processo de evolução e mudança. Transição é também compatível com as teorias que enfatizam adaptação.

Para TORRES (1986) e BLUE et al. (1989) a teoria de Roy aplica-se à pessoa, família, grupo ou comunidade considerando-os como sistemas adaptativos. As pessoas, neste mundo em mudança, precisam manter sua integridade e adaptar-se continuamente. A sua teoria procura responder a como as pessoas adaptam-se às mudanças a que estão sujeitas e propõe que a enfermagem busque contribuir à adaptação com o objetivo de promover a saúde, incentivando a pessoa a utilizar seus mecanismos de enfrentamento. Roy, conforme TORRES (1986), considera a pessoa como um ser biopsicossocial em interação constante com um ambiente em mudança que, ao transitar pelo continuum saúde-doença, encontrará problemas aos quais terá que se adaptar.

Como percebe-se, as transições incluem várias abordagens de teoristas que estão intimamente ligadas aos eventos que compõem o ciclo vital. Pela variedade de enfoques, a transição relaciona-se ao cuidado visando a prevenção e intervenção de enfermagem para cada caso específico de ocorrência, propiciando um desvelamento aos enfermeiros de um enfoque inovador e possibilitando a reafirmação de teorias já publicadas e também a proposição de novas teorias.

\section{IDÉIAS CONCLUSIVAS}

Os vários eventos que permeiam a vida das pessoas exigem respostas para que estas possam ultrapassar momentos de instabilidade, as mudanças de auto-estima, as mudanças de desempenho de papéis, a ansiedade, a depressão e a insegurança, para alcançar o equilíbrio perdido.
A enfermagem, ao realizar o cuidado baseado em um modelo de transição, estaria contribuindo para aumentar as possibilidades de ajuda ao indivíduo, não enfocando apenas a cura. O importante é fornecer suporte a uma pessoa em transição, auxiliando-a ao proteger e manter a sua saúde para o futuro. O objetivo da intervenção de enfermagem é cuidar dos clientes, criando condições condutivas a uma transição saudável, considerando o ser em mudança como um ser holístico, integral. As idéias e pesquisas surgidas, incluindo a prática, sobre o cuidado, podem ser consideradas como um processo de desenvolvimento e, portanto, transicional da profissão. Assim, os enfermeiros encorajados e inspirados em desenvolver seu trabalho alicerçado no cuidado, têm agora um novo enfoque a ser aplicado, o cuidado transicional - que é expressado em momentos específicos através de comportamentos particularizados.

$\mathrm{O}$ cuidado com este enfoque acrescenta ao que já é realizado, revitalizando o ensino, a assistência, a pesquisa em benefício do cliente, razão de ser da profissão do enfermeiro. O cuidado transicional humano traz respostas à valorização do ser uma vez que é o sujeito da ação do cuidado, não se limita a funções, papéis ou tarefas. O cuidado está ligado de alguma forma a cada estágio do desenvolvimento humano, favorece a maturidade, o crescimento em busca de maior equilíbrio e estabilidade.

O cuidado transicional não é algo definível, palpável, visível, não é algo que possa se reduzir a uma simples definição, mas ele surge da conscientização do enfermeiro ao desvelar a compreensão do cliente enquanto vivencia o processo transicional. A transição permeia todos os momentos da vida. Porém, estes momentos necessitam ser enfrentados através de comportamentos que possibilitem o cuidado particularizado a cada situação vivenciada, nas suas possibilidades de vir-a-ser consigo mesmo e com o outro. O cuidado transicional é o cuidado com a preocupação às etapas transicionais do ser, exposto a todas as suas possibilidades.

\section{TRANSITIONARY HUMAN CARE IN NURSING TRAJECTORY}

The present paper addresses human beings' transitionary events which permeate their vital cycle, as well as the conductive conditions to the transition processes and their care relations. It focuses on the several distinguishing types of transition and their conceptualizations throughout the scope of transitional care. Such care is concerned with the features of the events which derive change, as well as the individual variables and the context interacting with those features, facilitating the understanding of the interaction process and assimilation of the change. From that conceptualization, it targets the development of nursing interventions facing the transitional processes in the light of nursing theories, which can be re-stated in transition terms. Due to the variety of focuses, transition is related to nursing care and it aims at the prevention and intervention for each specific case, providing nurses with an innovative focus of care. It points out that care is somehow tied up to each developmental stage, favouring maturity and growth in the search for a larger balance and stability. Care facing transition brings about answers to the valuing of the being. 


\section{EL CUIDADO HUMANO TRANSICIONAL EN LA TRAYECTORIA DE ENFERMERÍA}

Éste trabajo aborda los eventos transitorios del ser humano que se desarrollan conjuntamente con el ciclo vital, así como las condiciones conductivas de los procesos de transición y sus relaciones con el cuidado. Enfatiza la distinción entre los varios tipos de transición y sus conceptualizaciones dentro de la dimensión del cuidado transitorio. El cuidado considera las características de los eventos que producen el cambio, así como variables individuales y de medios que interactuan con las características del evento, propicia la comprensión del proceso de interacción y asimilación del cambio. A partir de esta contextualización se orienta para el desarrollo de intervenciones de enfermería frente a los procesos transitorios por el empleo de teorías de enfermería, las cuales pueden ser reafirmadas en términos de transición. Por la variedad de enfoques la transición se relaciona al cuidado de enfermería, busca la prevención e intervención para cada caso específico de ocurrencia, propicia a los enfermeros un enfoque innovador de cuidado. Señala que el cuidado está conectado de alguna manera con cada estancia de desarrollo, favorece la madurez y el crecimiento en la búsqueda de mayor equilibrio y estabilidad. El cuidado en frente de la transición trae respuestas a la valoración del ser.

TÉRMINOS CLAVES: proceso del grupo, cuidado de enfermería

\section{REFERÊNCIAS BIBLIOGRÁFICAS}

01. BOEMER, M.R. Abordagem "do caring". Revista Paulista de Enfermagem, São Paulo, v. 4, n. 2, p. 55-58, abr/mai./jun.1984.

02. BLUE, C.L.; BRUBAKER, K.M.; PAPAZIAN, K.R.; RIESTER, C.M. Callista roy. modelo de adaptación. In: MARRINER, A. Modelos y teorías de enfermeria. Barcelona: Ediciones Rol, 1989. p. 260-274.

03. CAREY, E.T.; RASMUSSEN, L.; SEARCY, B.; STARK, N.L. Hildegard E. Peplau enfermería psicodinámica. In: MARRINER, Ann. Modelos y teorías de enfermeria. Barcelona: Ediciones Rol, 1989. p. 157-170.

04. CARTER, B.; McGOLDRICK, M. As mudanças no ciclo de vida familiar: uma estrutura para a terapia familiar. In: CARTER, B.; MCGOLDRICK, M. et al. As mudanças no ciclo de vida familiar: uma estrutura para a terapia familiar. Trad. Maria Adriana Veríssimo Veronese. 2. ed. Porto Alegre: Artes Médicas, 1995. p. 7-29.

05. CASTANHEL, M.S.Del; BOEHS, A.E. Cuidando de uma família na comunidade - uma experiência utilizando um referencial teórico. Texto e Contexto - Enfermagem, Florianópolis, v.2, n.2, p. 87-98, jul./dez. 1993.

06. CHICK, N.; MELEIS, A.I. Transitions: a nursing concern. In: CHINN, P. L. Nursing research methodology. Rockeville: Aspen, 1986. p. 237257.

07. CONNER, S.S.; WATT, J.K. D.E. Johnson: modelo de sistema de conducta. In: MARRINER, A. Modelos y teorías de enfermeria. Barcelona: Rol, 1989. p. 247-259.

08. DREW, N. Expansion and confirmation: a phenomenology of patients' experiences with caregivers. Image: Journal of Nursing Scholarship, Indianápolis, v. 18, n.2, p. 39-43, 1986.
09. ERDMANN, A.L. A complexibilidade no cotidiano de um sistema organizacional de cuidados de enfermagem. Florianópolis, 1995. Tese (Doutorado) - Universidade Federal de Santa Catarina.

10. FOLI, K.J.; JOHNSON, T.; MARRINER, A.; POAT, M.C.; POPPA, L.; ZORETICH, S.T. Myra Estrin Levine. Quatro princípios de conservación. In: MARRINER, A. Modelos y teorías de enfermeria. Barcelona: Ediciones Rol, 1989. p. 293-301.

11. FRIEDMAN, E.H. Sistemas e cerimônias: uma visão familiar dos ritos de passagem. In: CARTER, B.; McGOLDRICK, M. et al. As mudanças no ciclo da vida familiar: uma estrutura para a terapia familiar. Trad. Maria Adriana Veríssimo Veronese. 2. ed. Porto Alegre: Artes Médicas, 1995. p. 106-130.

12. GRIFFIN, A.P. A phylosophical analysis of caring in nursing. Journal of Advanced Nursing, Oxford, v. 8, p. 289-295, 1983.

13. HALL, B.A. The change paradigm in nursing: growth nursing persistence. Advances in Nursing Science, Mariland, v.3, n.4, p. 1-6, 1981.

14. HOBBLE, W.H.; LANSINGER, T.J.T. Modelo de relación humano a humano. In: MARRINER, A. Modelos y teorías de enfermeria. Barcelona: Rol, 1989. p. 171-178.

15. IMBER-BLACK, E. Transições idiossincráticas do ciclo de vida e rituais terapêuticos. In: CARTER, B.; McGOLDRICK, M. et al. As mudanças no ciclo de vida familiar: uma estrutura para a terapia familiar. Trad. Maria Adriana Veríssimo Veronese. 2. ed. Porto Alegre: Artes Médicas, 1995, p. 131-143.

16. LACERDA, M.R. O cuidado transpessoal de enfermagem no contexto domiciliar. Florianópolis, 1996. Dissertação (Mestrado) Universidade Federal de Santa Catarina. 
17. MAJEWSKI, J.L. Conflicts, satisfactions, and attitudes during transitions to the maternal role. Nursing Research, New York, v. 35, n. 1, p. 1014, Jan./Feb. 1986.

18. McGOLDRICK, M. A união das famílias através do casamento: o novo casal. In: CARTER, B.; McGOLDRICK, M. et al. As mudanças no ciclo de vida familiar: uma estrutura para a terapia familiar. Trad. Maria Adriana Veríssimo Veronese. 2. ed. Porto Alegre: Artes Médicas, 1995, p. 184-205.

19. MERCER, R.T.; NICHOLS, E.G.; DOYLE, G.C. Transitions over the life cycle: A comparison of mothers and non mothers. Nursing Research, New York, v. 37, n. 3, p. 144-151, May/June 1988.

20. MORSE, J.M.; SOLBERG, S.M.; NEANDER, W.L.; BOTTORFF, J.L.; JOHNSON, J.L. Concepts of caring and caring as a concept. Advances in Nursing Sciences, Maryland, v. 13, n. 1, p. 1-14, 1990.

21. MORSE, J.M.; BOTTORFF, J.; NEANDER, W.; SOLBERG, S. Comparative analysis of conceptualizations and theories of caring. Image: Journal of Nursing Scholarship, Indianápolis, v.23, n. 2, p. 119-126, 1991.

22. MURPHY, S.A. Human responses to transitions: a holistic nursing perspective. Holistic Nursing Practice, v. 4, n. 3, p. 1-7, 1990.

23. NEVES-ARRUDA, E.; SILVA, A.L.de. Cuidando confortando: um programa emergente de pesquisa em enfermagem. Texto e Contexto Enfermagem, Florianópolis, v. 3, n. 1, p. 116127, jan./jun 1994.

24. NUNES, A.M.P.; NEVES-ARRUDA, E. Repensando conforto no processo de cuidar em enfermagem. Texto e Contexto - Enfermagem, Florianópolis, v. 4, n. 1, p. 139-149, jan./jun. 1995.

25. NUNES, D.M. Linguagem do cuidado. São Paulo, 1995. Tese (Doutorado) - Universidade Federal de São Paulo.

26. PATRICIO, Z.M. Promovendo a cidadania através do conceito cuidado. Texto e Contexto Enfermagem, Florianópolis, v. 1, n. 1, p. 89-106, jan./jun. 1992.

27. PATRICIO, Z.M. O processo de trabalho da enfermagem frente às novas concepções de saúde: repensando o cuidado/propondo o cuidado (holístico). Texto e Contexto - Enfermagem, Florianópolis, v.2, n. 1, p. 67-81, jan./jun. 1993.
28. RADÜNZ, V. Cuidando e se cuidando: fortalecendo o "self" do cliente oncológico e o "self" da enfermeira. Florianópolis, 1994. Dissertação (Mestrado) - Universidade Federal de Santa Catarina.

29. ROACH, S.M.S. The human act of caring: a blueprint for the health profissions. Ottawa: Canadian Hospital Associations, 1993. 148p.

30. SANTOS, V.dos. Buscando um referencial de cuidados integrativos, na era do marketing e comunicação. Texto e Contexto - Enfermagem, Florianópolis, v. 2, n. 2, p. 76-86, jul./dez. 1993.

31. SCHUMACHER, K.L.; MELEIS, A.I. Transitions: a central concept in nursing. Image: Journal of Nursing Scholarship, Indianápolis, v. 26, n. 2, p. 119-127, 1994.

32. SILVA, A.L.da. O cuidado no encontro de quem cuida e de quem é cuidado. Florianópolis, 1996. Concurso Titular - Universidade Federal de Santa Catarina.

33. SILVA, A.L.da; BORENSTEIN, M.S. Ser e viver saudável no mundo: buscando novos caminhos no cuidar pesquisando com o ser - doente. Texto e Contexto - Enfermagem, Florianópolis, v. 1, n. 2, p. 56-69, jul./dez. 1992.

34. SOUZA, A.I.J.de. No cuidado com os cuidadores em busca de um referencial para a ação de enfermagem oncológica pediátrica fundamentada em Paulo Freire. Florianópolis, 1995. Dissertação (Mestrado) - Universidade Federal de Santa Catarina.

35. TOMLINSON, P.S. Spousal differences in marital satisfaction during transition to parenthood. Nursing Research, New York, v. 36, n. 4, p. 239243, Jul./Aug. 1987.

36. TORRES, G.. Theoretical foundations of nursing. Norwalk, Connecticut: Appleton - Century Crofts, 1986.

37. WALDOW, V.R. Cuidado: uma revisão teórica. Revista Gaúcha de Enfermagem, Porto Alegre, v. 13 , n. 2, p. 29-35, 1992.

38. WALDOW, V.R. Cuidar/Cuidado: O domínio unificador da enfermagem. In: WALDOW, V.R.; LOPES, M.J.M.; MEYER, D.E. Maneiras de cuidar, maneiras de ensinar: a enfermagem entre a escola e a prática profissional. Porto Alegre: Artes Médicas, 1995. p. 7-30.

39. WATSON, J. Nursing: human science and human care. Norwalk, Connecticut: Appleton - Century - Crofts, 1985. 